\title{
Acknowledgment to reviewers for 2018
}

On behalf of the editor and editorial board, we would like to express our sincere gratitude for the reviewers and contributors who have generously endowed with their time and efforts in appraising the manuscripts submitted to Clinical and Experimental Reproductive Medicine in 2018.

It was their help that made it possible for the journal to achieve a new level of caliber and rigor.

\begin{tabular}{|c|c|c|c|}
\hline Cha, Jeong-Ho & Agaon Fertility Clinic & Kim, Seul Ki & Seoul National University \\
\hline Cha, Sun Hwa & Maria Fertility Center & Kim, Yong-Jin & Korea University \\
\hline Chang, Eunmi & CHA University & Ko, Chemyong & University of Illinois at Urbana-Champaign, \\
\hline Cheon, Yong-Pil & Sungshin Women's University & & College of Veterinary Medicine \\
\hline Cho, Hyun Hee & The Catholic University of Korea & Koo, Hwa Seon & CHA University \\
\hline Cho, Moon-Kyoung & Chonnam National University & Ku, Seung-Yup & Seoul National University \\
\hline Cho, Sihyun & Yonsei University & Lee, Dong Ryul & CHA University \\
\hline Cho, Youn Jin & Dong-A university & Lee, Heejun & CHA University \\
\hline Choi, Jung Kyu & CHA University & Lee, Hee Sun & Seoul Rachel Fertility center \\
\hline Choi, Won-Jun & Gyeongsang National University & Lee, Hyo Serk & JTS arology center \\
\hline Choi, Young Sik & Yonsei University & Lee, Jaeho & CHAIVF Seoul Station Center \\
\hline Choi, Youngsok & CHA University & Lee, Jaewang & Eulji University \\
\hline Chun, Sang-Young & Chonnam National University & Lee, Joongyeup & Hamchoon Women's Clinic \\
\hline Chun, Sungwook & Inje University & Lee, Jung Ryeol & Seoul National University \\
\hline Chung, Mikyung & Seoul Rachel Fertility Center & Lee, Sang Min & Korea University \\
\hline Eum, Jin Hee & CHA Fertility Center & Lee, Sung Ho & Sangmyung University \\
\hline Gye, Myung Chan & Hanyang University & Lee, Sung Ki & Konyang University \\
\hline Hong, In Seon & Gachon University & Lim, Chun Kyu & CHA Fertility Center \\
\hline Hong, Seung Hwa & Chungbuk University & Lim, Hyunjung & Konkuk University \\
\hline Joo, Bo-Sun & Good Moonhwa Hospital & Lim, Kyoungtaek & Maria Hospital \\
\hline Joo, Jong Kil & Pusan National University & Park, Dong-Wook & Dankook University \\
\hline Jun, Jin Hyun & Eulji University & Park, Hyun Jun & Pusan National University \\
\hline Jung, Jae Hung & Yonsei University & Park, Hyun Tae & Korea University \\
\hline Kim, Chunghyon & Hamchoon Women'S Clinic & Park, Joon Cheol & Keimyung University \\
\hline Kim, Dae Keun & CHA University & Park, Yong-Seog & Cheil General Hospital \& Women's Healthcare Center \\
\hline Kim, Dong Suk & CHA University & Ruy, Sangwoo & CHA University \\
\hline Kim, Gi Jin & CHA University & Song, Haengseok & CHA University \\
\hline Kim, Gi Young & MizmediHospital & Song, Seung Hun & CHA University \\
\hline Kim, Hoon & Seoul National University & Yang, Kwang Moon & Maria Fertility Hospital \\
\hline Kim, Hye Ok & CHA University & Yoon, Sook-Young & CHA University \\
\hline Kim, Jin Ju & Seoul National University & Youm, Hyewon & Seoul National University Bundang Hospital \\
\hline Kim, Miran & Ajou University & Yun, Bo Hyon & Yonsei University \\
\hline Kim, Nam Keun & CHA University & & \\
\hline
\end{tabular}

This is an Open Access article distributed under the terms of the Creative Commons Attribution Non-Commercial License (http://creativecommons.org/licenses/by-nc/4.0/) which permits unrestricted non-commercial use, distribution, and reproduction in any medium, provided the original work is properly cited. 